Proc. XIX International School of Semiconducting Compounds, Jaszowiec 1990.

\title{
TWO CHARGE STATES OF SULPHUR IN GaSb*
}

\author{
L. DobaczewsKi, \\ Institute of Physics, Polish Academy of Sciences, Al. Lotników 32/46, 02-668 \\ Warszawa, Poland \\ University of Manchester, Institute of Science and Technology, Electrical Engineering \\ and Electronics Department, Manchester M60 1QD, PO Box 88, United Kingdom

\section{P. KACZOR, G. KARCZEWSKI} \\ Institute of Physics, Polish Academy of Sciences, Al. Lotników 32/46, 02-668 \\ Warszawa, Poland

\section{AND I. POOLE} \\ University of Manchester, Institute of Science and Technology, \\ Electrical Engineering and Electronics Department, Manchester M60 1QD, PO Box 88, \\ United Kingdom \\ (Received August 8, 1990) \\ Results of the isothermal DLTS, photoconductivity, and photo-Hall mea- \\ surements, showed that the sulphur-related center in GaSb, being a DX-type \\ defect, has two charge states. \\ PACS numbers: 71.55.Eq, 78.50.Ge, 79.70.+q
}

Such anomalous effects as metastability and persistent photoconductivity at temperatures below $80 \mathrm{~K}$ were found for sulphur in $\mathrm{GaSb}$ already in the early seventies [1]. Dmowski et al. [2] gave the explanation for this peculiar behavior based on a large lattice relaxation model. Experimental data in the present work allow to reexamine the previous models and consider the sulphur defect as belonging to the so-called DX centres which, according to recent experimental [3] and theoretical [4] indications, appear to have two charge states and form a negative- $U$ system [5].

*This work has been supported by the program CPBP 01.05 in Poland, and by the Science and Engineering Research Council in the UK. 
The isothermal capacitance transients, photocurrent response and photoionization process were measured on S-doped MBE-grown $\mathrm{GaSb}$-samples with in situ deposited Al-Schottky contacts. The concentration of electrically active sulphur center in the sample was $5 \times 10^{17} \mathrm{~cm}^{-3}$, more than an order of magnitude larger than the residual acceptor concentration [6].

As the parameters describing the thermal emission process for the DX centers in binary semiconductors are not broadened due to alloy fluctuations the analysis of the transients could be made in a straightforward way. All of the transients could be decomposed with great accuracy into a sum of two monoexponentials:

$$
\Delta C / \Delta C_{0}=N_{1} \exp \left(-e_{1} t\right)+N_{2} \exp \left(-e_{2} t\right)
$$

with comparable amplitudes $N_{1}, N_{2}$ which indicates the existence of two energy levels of the defect. The Arrhenius plot of the emission rates $e_{1}$ and $e_{2}$ inferred from the fitting is shown in Fig. 1. Standard DLTS experiments were not able

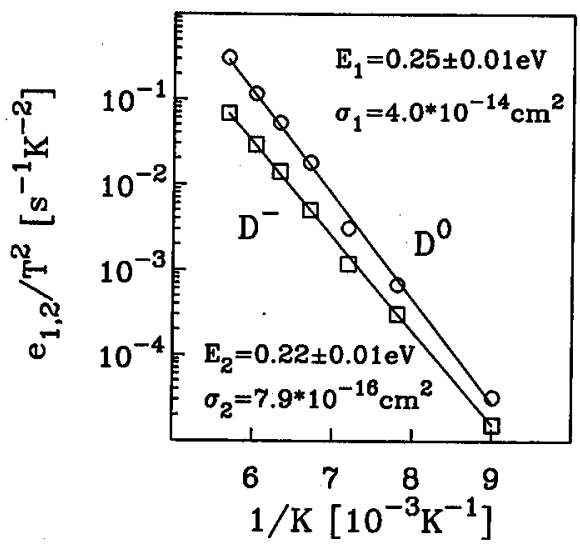

Fig. 1. Arrhenius plot of the two emission rates inferred from the fitting of the two-exponential function to the capacitance transients.

to resolve both emission rates because their temperature dependencies are very similar (small difference in thermal emission energies) - the resulting peak was simply broadened. The capture cross-sections of the two energy states differ almost by two orders of magnitude suggesting that the states have different charges.

These observations were confirmed by a careful analysis of the amplitudes of the transients as a function of the filling pulse width (Fig. 2). The experimental points were fitted assuming trapping kinetics inferred from the rate equations for an initially empty two-electron center. Only two fitting parameters were used: $c_{1}$ and $c_{2}$ - the capture rates attributed to the single and double charged states, respectively. It can be clearly seen that at lower temperature one of the charge states becomes thermodynamically unstable (curve $N_{1}\left(t_{f i l l}\right)$ ), though it can bind -an electron for a certain period of time. It suggests that the energy state denoted by 1 is an intermediate state for the capture process. 


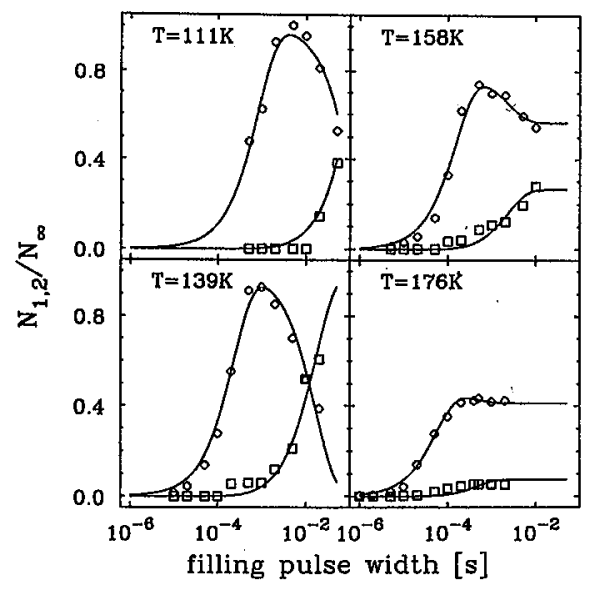

Fig. 2. Amplitudes $N_{1}$ (circles), $N_{2}$ (squares) as a function of the filling pulse width. The solid curves are the best fit to the experimental points, where the trapping kinetics was inferred from the rate equations for initially empty two-electron center.

Temperature dependence of the ratio $c_{1} / c_{2}$ revealed that the capture barrier (if any) for the state with a larger capture cross-section is about $60 \mathrm{meV}$ smaller than the other one. Consequently, the already observed metastability effects seem to be a feature of the doubly charged center rather than of the singly one.

The results of the photocapacitance experiments confirm the metastable nature of the center. After the photocapacitance transient was recorded only a relatively small and fast recapture could be observed, thus, the initial and final states of the sample seem to be nonequivalent. The initial conditions could be reestablished only through heating the sample up to a high temperature $(\approx 300 \mathrm{~K})$.

When the occupancy equilibrium between two sulphur states and the conduction band was disturbed by turning the light on and off overshoots in conductivity were observed; i.e. when the light was switched on, the relatively fast rise of the photocurrent was followed by a decrease to a value slightly larger than the dark current. Photo-Hall measurements have shown that the equilibrium free electron concentration drops below its 'dark' value but the mobility rises suggesting that the charge state of the centers responsible for the photocurrent changes. This overshoot- behavior is characteristic for a very strong compensation regime which, in our case, is possible only when the ground state of sulphur is an acceptor.

A variety of phenomenological models (e.g. strong compensation due to native acceptors, double donor, a single charged center with a long lived excited state) were considered in order to explain the data measured. But only the assumption of two charge states and negative- $\mathrm{U}$ character of the sulphur center in $\mathrm{GaSb}$ yields the 
consistent explanation of all experimental facts mentioned above. Detailed analysis of the equilibrium occupancy as a function of the temperature also confirms this hypothesis. However, the large entropy factor of both energy states cannot be explained without a convincing microscopic model of the formation of a negative- $U$ system in this case.

\section{References}

[1] A.I. Vul, A.I. Shik, Fiz. Tekh. Poluprovodn. 8, 1952 (1974).

[2] L. Dmowski, M. Baj, M. Kubalski, R. Piotrzkowski, S. Porowski, in Proc. 9-th Internat. Conf. Phys. Semicond., Edinburgh 1978, Inst. Phys. Conf. Ser. No 43, ed. B.L.H. Wilson, IOP, Bristol and London 1979, p. 417.

[3] L. Dobaczewski, P. Kaczor, J.M. Langer,to be published.

[4] D.J. Chadi, K.J. Chang, Phys. Rev. B 39, 10063 (1989).

[5] G.D. Watkins, Festkoerperprobleme Advances in Solid State Physics, Vol. 24, ed. P. Grosse, Vieweg, Braunschweig, 1984, p.163.

[6] I. Poole, M.E. Lee, K.E. Singer, J.E.F. Frost, T.M. Kerr, C.E.C. Wood, D.A. Andrews, W.J.M. Rothwell, G.J. Davies, J. Appl. Phys. 63,395 (1988). 